\title{
Hopkins scientist tunes in to treat tuberculosis
}

If biotic moxifloxacin will be the first new treatment for tuberculosis (TB) in more than four decades. And Chaisson is not a man who is used to failure.

An infectious disease expert and professor of medicine, epidemiology, and international health at the Johns Hopkins University School of Medicine, Chaisson is now leading two global studies to test the effectiveness of moxifloxacin to treat TB. The drug is already approved in the US and dozens of other countries to treat various respiratory infections.

Chaisson's analyses are part of a series of studies on moxifloxacin coordinated by the Global Alliance for TB Drug Development. Data will be collected from 2,500 patients in the US, Canada, Brazil, Spain, South Africa, and Uganda to assess the ability of moxifloxacin to shorten the treatment period required to cure the disease.

$\mathrm{TB}$ is a highly communicable bacterial disease that is the leading cause of death in HIV/ AIDS patients. More than 2 billion people are infected with the organism, and over the next 15 years TB disease will afflict an estimated 140 million people, killing 35 million of them. Current TB treatment is usually supervised by a caregiver and consists of a regimen of four antibiotic drugs that must be taken many times a day for six to eight months. Although this course of therapy is successful 95 percent of the time, the long period of drug use is problematic for many patients, who often miss doses and thereby decrease the drugs' efficacy. Substituting moxifloxacin for one of the four antibiotic drugs currently used could shorten the treatment period by many months, increasing the success of treatment while also decreasing the cost.

The JCI spoke to Chaisson about this new project, the future of TB therapy, and his other ambitious endeavors.

JCI: Why is TB such an enormous public health problem?

Chaisson: Despite having had drugs to treat and prevent TB for 50 years and a vaccine for 80 years, TB still kills 2 million people per year, mostly in developing countries. The current vaccine is clearly inadequate, and the current drugs require at least six months of treatment, posing a huge challenge to health systems around the world. The incidence of TB is increasing each year, with 9 million cases last year, and a large proportion of patients are not diagnosed and treated. In addition, there are about 300,000 cases per year of multi-drug-resistant $\mathrm{TB}$, which is exceedingly difficult to treat. Thus, TB is an enormous burden on developing countries and an important problem in the developed world.

JCI: What is currently used to treat TB worldwide, and why hasn't there been a new approach for four decades?

Chaisson: The four primary drugs for $\mathrm{TB}$ were all developed in a 25 -year period from the mid-1940s to the late 1960s. During that time, TB went from being an incurable, highly fatal disease to one that could be cured with less than a year of treatment. People felt that this was such an enormous scientific accomplishment that the problem was considered solved. Unfortunately, six months is still too long for many patients, and drug resistance has emerged. So a renewal of interest in developing TB drugs has sprung up in the past 5 years.

JCI: What exactly is moxifloxacin and how does it work?

Chaisson: Moxifloxacin is an advanced fluoroquinolone antibiotic that targets DNA gyrase and kills bacteria. In our center [the Johns Hopkins Center for TB Research], the drug has shown unusual potency against TB, particularly when combined with several of the older TB drugs. Studies in the mouse suggest that moxifloxacin could shorten the duration of TB treatment from six months to only three

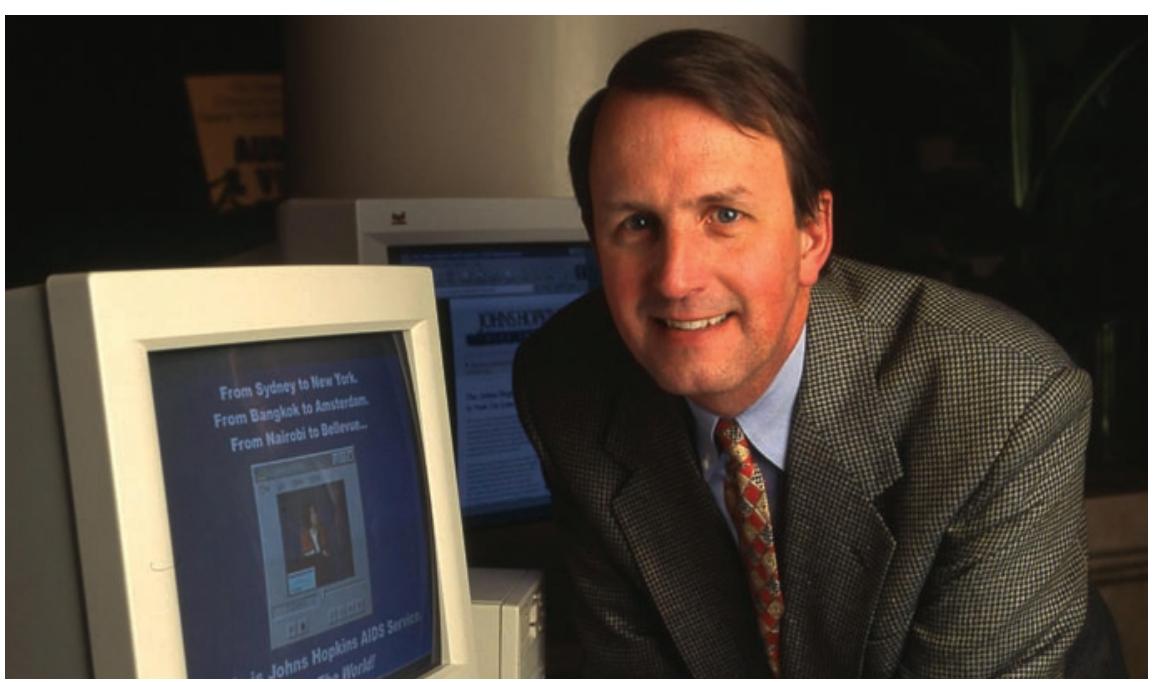

Richard Chaisson is leading two global studies that could change the way TB is treated. or four months, a major improvement that will promote public health.

JCI: How did you get involved in this field of research?

Chaisson: I am an infectious disease specialist with training in epidemiology. During my fellowship at UCSF I worked on HIV natural history but moonlighted in the TB clinic for fun and have always had an interest in the overlap of TB and HIV. I ran the Johns Hopkins AIDS Service from 1988-1998, and then in 1998 I founded the Johns Hopkins Center clinical scientists to attack TB in a coordinated fashion. My own work focuses on trials of treatment and prevention and public health interventions to control HIV-related TB.

JCI: What is your day-to-day life like? Times crossword puzzle, in the belief it will ward off dementia. I don't actually work in the lab, but run the center and clinical trials in the US, Brazil, and South Africa. I spend a lot of time communicating with collaborators, working with regulatory agencies and institutional review boards, and managing day-to-day scientific issues, such as the management of adverse events. I travel to study sites quite a bit, meet with collaborators, and try to analyze and publish results as quickly as possible. I maintain a clinic twice a month seeing HIV patients, and I attend on the inpatient HIV ward for one month each year.

JCI: What do you hope results from this study? for TB Research to bring together bench and

Chaisson: My day begins with the New York 
Chaisson: We hope the first study will show that moxifloxacin speeds up the sterilization time in sputum samples from TB patients, and then we hope to show that a regimen given for four months is as effective as one given for six months. We will also continue to look at the other drugs in the pipeline and make adjustments to the regimens as the trials proceed.

JCI: What do you consider to be your greatest scientific achievement?

Chaisson: I have made some contributions that have been worthwhile, I hope. Early on, I was able to show the importance of the interaction of TB and HIV in San Francisco. Over the past few years we have built on this work and explored the public health dimensions of TB and HIV, developing new models of TB control in the era of HIV. I was able to show that supervised treatment for TB improved survival and led to reduced incidence in Baltimore. And back when I was largely focused on HIV, my colleague Richard Moore and I pioneered the use of HIV clinical cohorts, which has led to numerous important observations on the natural history and treatment outcomes of HIV infection and played a critical role in developing treatment guidelines globally.

JCI: What about your greatest accomplishment in life outside of science?

Chaisson: My greatest non-work-related accomplishment is having a wonderful family, for which I can only take a small amount of credit. I was lucky enough to marry a wonderful woman, Judy Harding, and we have two teenage daughters who give us nothing but joy.

JCI: In addition to the moxifloxacin study, you also direct the Consortium to Respond Effectively to the AIDS/TB Epidemic (CREATE), an international effort to treat and control the spread of tuberculosis in countries hit hardest by the two epidemics. What has been your most interesting experience in that realm?

Chaisson: The work I am doing on CREATE has drawn the attention and support of some very important people, not the least of whom are Nelson Mandela and Desmond Tutu. Mr. Mandela attended the launch of CREATE in Bangkok last year, and gave one of the most important TB advocacy speeches in history. Archbishop Tutu attended our last investigators' meeting in Cape Town in September and gave the most moving speech I have heard in years.

\section{Stacie Bloom}

\section{Taking the direct route to make open access even easier}

F entific nonprofit publishers in the US have offered the NIH direct links to their journals to make research access even easier for the public, and at no cost to boot.

Their proposal, sent to NIH director Elias Zerhouni on October 25, laid out a plan for the NIH to provide access to their journal websites using links from abstracts that are already in place and indexed on Medline. This system would bring the NIH a step closer to their known goal of complete public access to all NIH-funded research. Currently, the $\mathrm{NIH}$ requests that all grantees submit their manuscripts as soon as they have been accepted for publication, before they have benefited from production changes such as copy editing and layout.

A major concern has been the potential of confusion if more than one version of the manuscript is made available, which could lead to errors. The new proposal would eliminate this worry - the publishers are offering the finalized manuscripts to the NIH at no charge so that only the published version of the paper would be available, keeping the scientific record intact while maintaining copyright protections. This arrangement would also eliminate the need to create a taxpayerfunded infrastructure that would support a web database to publish and house the penultimate versions of the papers and will permit seamless linkage to more than 1 million scientific articles, a number that will increase by approximately 15,000 each month.

"Overnight and at no cost to taxpayers, this proposal will make it easy for the public to access vast amounts of the most accurate scientific and medical information available," said Chris Lynch, vice president of publishing for the Massachusetts Medical Society, in a press release. "What we are proposing is for the NIH to become the public's doorway into the universe of research that non-profit publishers already provide to the public everyday."

"This is simply another step in fostering collaboration among the scientific communities and the public," said Lenne P. Miller of The Endocrine Society. "We look forward to hearing back from Director Zerhouni and to meeting with him to move forward on an exciting partnership that would deliver enormous public benefits for generations to come."

\section{SB}

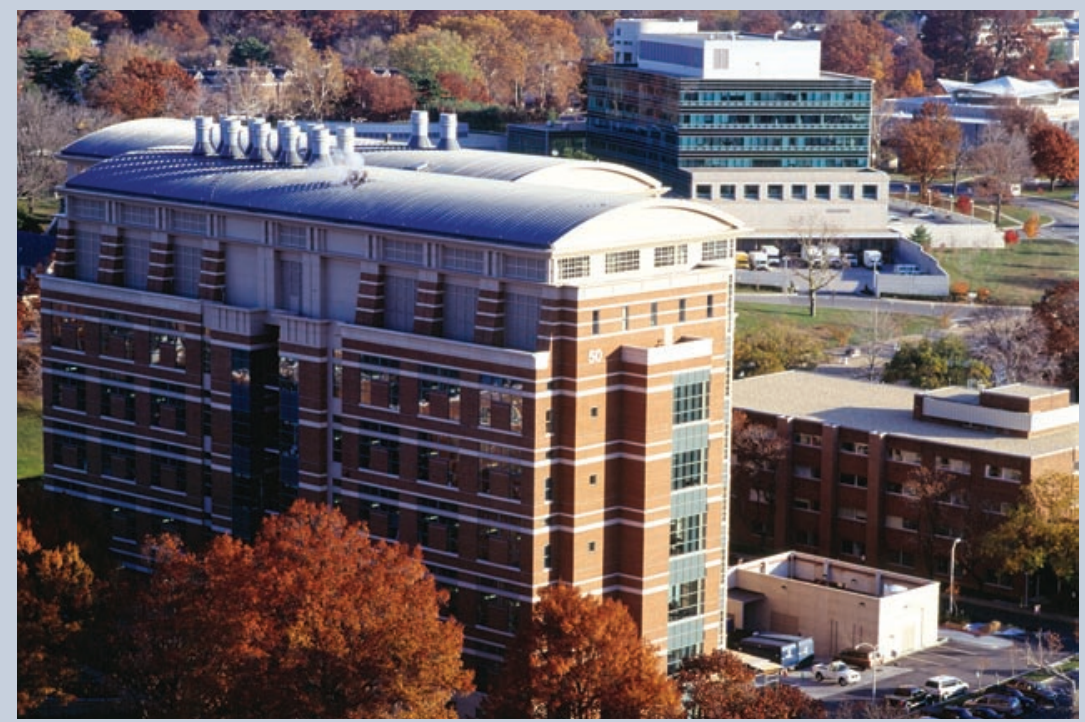

To foster easy public access, a leading group of nonprofit publishers have offered the $\mathrm{NIH}$ direct links to their journals. 